\title{
Topological Quantum Liquids with Long-Range Couplings
}

\author{
Kristian Patrick, ${ }^{1, *}$ Titus Neupert, ${ }^{2}$ and Jiannis K. Pachos ${ }^{1}$ \\ ${ }^{1}$ School of Physics and Astronomy, University of Leeds, Leeds LS2 9JT, United Kingdom \\ ${ }^{2}$ Department of Physics, University of Zurich, Winterthurerstrasse 190, CH-8057 Zurich, Switzerland \\ (Received 28 November 2016; revised manuscript received 24 February 2017; published 29 June 2017) \\ Very few topological systems with long-range couplings have been considered so far due to our lack of \\ analytic approaches. Here we extend the Kitaev chain, a 1D quantum liquid, to infinite-range couplings and \\ study its topological properties. We demonstrate that, even though topological phases are intimately linked \\ to the notion of locality, the infinite-range couplings give rise to topological zero and nonzero energy \\ Majorana end modes depending on the boundary conditions of the system. We show that the analytically \\ derived properties are to a large degree stable against modifications to decaying long-range couplings. Our \\ work opens new frontiers for topological states of matter that are relevant to current experiments, where \\ systems with interactions of variable range can be designed.
}

DOI: 10.1103/PhysRevLett.118.267002

Introduction.-Traditionally, physical systems are modeled by Hamiltonians that are local. This condition is linked to several quantum properties, such as the entropic area law behavior or the existence of local order parameters. Nevertheless, models with nonlocal couplings become increasingly relevant in describing experiments, with Rydberg atoms [1], trapped ions [2], or atoms in optical cavities [3], that naturally host long range interactions. Moreover, variational Ansätze employed to model interacting systems have so far been local. While these Ansätze have been successful in explaining important effects of interacting fermions $[4,5]$, our limitation in understanding the majority of strongly correlated electron systems may be due to the intrinsic nonlocal nature of Coulomb interactions.

Recognizing this gap in our knowledge, an increasing number of studies have recently been focused on nonlocal models [6-9], including Dirac [10-19] and Majorana [20-29] fermion chains. In these cases, nonlocality is defined as there being no integer $n$ such that couplings and interactions have support over $n$ sites in the thermodynamic limit. Because of the complexity of nonlocal systems, these studies mainly rely on numerical investigations. However, conceptual understanding often requires analytical solutions. Here we address the question, how can a system with nonlocal couplings be characterized topologically? Indeed, infiniterange couplings cause a meltdown of the concepts of locality and dimensionality that seem to be necessary for identifying a topological phase. Moreover, a recent study of the nonlocal Kitaev chain [24] showed that localized end states may

Published by the American Physical Society under the terms of the Creative Commons Attribution 4.0 International license. Further distribution of this work must maintain attribution to the author(s) and the published article's title, journal citation, and DOI. acquire nonzero energy, and that the topological invariant of the short-ranged model may change or completely lose its quantization when long-range couplings are added.

To conclusively address this problem we analytically investigate Kitaev's chain [30] decorated with infinite-range tunneling and pairing couplings. To understand the properties of the end modes we need to consider two distinct geometries, a semi-infinite chain and a finite chain. Via a generating function method, we analytically demonstrate that the semiinfinite chain exhibits a single localized zero energy end mode. This Majorana zero mode is topologically stable in the same way as the edge modes of the local Kitaev chain. Moreover, its presence is in agreement with the nontrivial value of the Pfaffian topological invariant of the model, that is generalized here to open chains, thus demonstrating the persistence of topological properties in the presence of infinite-range couplings. For the physically relevant case of a finite chain both end states hybridize due to the nonlocal couplings and acquire nonzero energy. Analytical expressions for the energy eigenvalues are found along with the exact form of the end modes, providing a theoretical framework for investigating localization properties of other long-range models. Still, Majorana zero modes can be identified in the entanglement spectra encoded in the quantum correlations of an appropriately partitioned finite chain. Finally, we demonstrate the stability of our findings when the infiniterange couplings are allowed to decay. Thus, the infiniterange model shares the same topological properties as the short-range one. Note that, in the infinite range limit any attempt to close the chain results in cancellations of longrange terms. To overcome this problem we choose to restrict ourselves to an open chain, and aim to derive all of the topological properties that are present.

The nonlocal Majorana model.-We start from Kitaev's superconducting chain [30] with $N$ sites, where we take the tunneling and pairing terms to act between all pairs of 
fermions. The nonlocal superconducting Hamiltonian with open boundary conditions reads

$H=\sum_{j=1}^{N}\left[\sum_{l=1}^{N-j}\left(\frac{J}{l^{\alpha}} a_{j}^{\dagger} a_{j+l}+\frac{\Delta}{l^{\alpha}} a_{j} a_{j+l}\right)+\frac{\mu}{2} a_{j}^{\dagger} a_{j}\right]+$ H.c.,

where $a_{j}$ and $a_{j}^{\dagger}$, with $j=1, \ldots, N$, are fermionic operators, $J$ is a positive tunneling coupling, $\Delta=|\Delta| e^{i \theta}$ is the superconducting coupling, and $\mu$ is the local chemical potential. The parameter $\alpha$ controls the range of couplings. The case $\alpha \rightarrow \infty$ corresponds to the well-known local Kitaev chain. The $\alpha=0$ case corresponds to the nondecaying chain with infinite-range couplings, as shown in Fig. 1 (top). We initially consider the $\alpha=0$ case, before we turn to the power law behavior with $\alpha \neq 0$. The infinite-range model can be written, via the Jordan-Wigner transformation, as a spin chain with infinite-range cluster interactions $[19,31,32]$.

To gain an insight to the behavior of the system we decompose the Dirac fermions into Majorana operators, as $a_{j}=e^{-i(\theta / 2)}\left(\gamma_{2 j-1}+i \gamma_{2 j}\right)$. This transforms Hamiltonian Eq. (1) into a Majorana chain with infinite-range couplings, as shown in Fig. 1 (bottom). For the choice of couplings $\mu=0$ and $J= \pm|\Delta|$, zero energy modes emerge, localized at the end sites of the chain, much in the same way as the local Majorana Hamiltonian [30].

To analytically determine the energy and localization properties of the Majorana end states for general values of the couplings $\mu, J$, and $\Delta$, we need to construct a systematic method. Our approach is based on the generating function that is routinely used to probe edge characteristics of local models [33-35]. Assume the state $|\psi\rangle=\sum_{j=1}^{N} \chi_{j}^{\dagger} \psi_{j}|0\rangle$ is an eigenstate of Hamiltonian (1) with energy $E$, where $\chi_{j}^{\dagger}=\left(\begin{array}{ll}a_{j}^{\dagger} & a_{j}\end{array}\right), \psi_{j}=\left(\begin{array}{l}\psi_{j}^{A} \\ \psi_{j}^{B}\end{array}\right)$ is the amplitude of the state at site $j$ and $|0\rangle$ is the superconducting vacuum. The equation $H|\psi\rangle=E|\psi\rangle$ leads to the recursion relation

$$
\sum_{l=1}^{N}\left[\Gamma_{2} \psi_{j+l}+\Gamma_{2}^{\dagger} \psi_{j-l}\right]+\left(\Gamma_{1}-2 E\right) \psi_{j}=0,
$$

where $\Gamma_{1}$ and $\Gamma_{2}$ contain the coupling constants $J, \Delta$, and $\mu$. By defining $\Psi_{j}=\sum_{k=j}^{N} \psi_{k}$, Eq. (2) takes the simpler form $M \Psi_{j}+K \Psi_{j+1}+\Gamma_{2}^{\dagger} \Psi_{1}=0$, where $M=\Gamma_{1}-\Gamma_{2}^{\dagger}-2 E$ and $K=\Gamma_{2}-\Gamma_{1}+2 E$. Multiplying this by $z^{j}$ for $z \in \mathbb{C}$, summing over all $j$, and defining the generating function as

$$
G(z)=\sum_{j=1}^{N} z^{j-1} \Psi_{j}
$$

we obtain an equation for $G(z)$ that can be directly solved to give

$$
G(z)=(K+z M)^{-1}\left(K-\Gamma_{2}^{\dagger} \frac{z\left(1-z^{N}\right)}{1-z}\right) \Psi_{1} .
$$
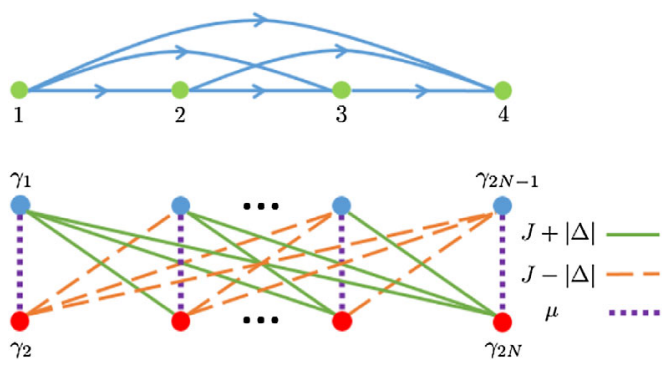

FIG. 1. (Top) Kitaev's chain of size $N=4$ with infinite-range couplings. While tunneling couplings create a fully connected graph, the pairing couplings encode direction that preserves the one-dimensional character of the model. (Bottom) The infiniterange Kitaev chain in terms of Majorana operators, $\gamma_{2 j-1}$ (blue) and $\gamma_{2 j}$ (red). The Majoranas are coupled with strengths $J+|\Delta|$ (green), $J-|\Delta|$ (orange), and $\mu$ (purple).

For concreteness, we take $\Psi_{1}=\left(\begin{array}{c}\phi_{1} \\ \phi_{2}\end{array}\right)$, where $\phi_{1}$ and $\phi_{2}$ are to be determined.

From the definition of $G(z)$ given in Eq. (3) we see that the properties of its poles depend on the distribution of the amplitudes $\psi_{j}$ of the modes along the chain. The closed form, Eq. (4), allows us to study the poles of $G(z)$. Apart from the explicit $z=1$ pole, the rest can be produced by the inverse matrix $(K+z M)^{-1}$. For that we consider the zeros $\left\{z_{1}, z_{2}\right\}$ of $\operatorname{det}(K+z M)=\left[4 E^{2}-(J-\mu)^{2}\right](1-z)^{2}+$ $|\Delta|^{2}(1+z)^{2}$ that are not canceled from the rest of the expression. Vieta's formula relates the zeros of $\operatorname{det}(K+z M)$ with the ratio of the coefficients of $z^{0}$ and of $z^{2}$, giving $z_{1} z_{2}=1$ [36]. As zeros of the determinant with $z_{i}=1$ correspond to bulk modes, we are interested in the zeros $\left\{z_{1}, z_{2}\right\}$ that have amplitudes larger or smaller than one (for more details see Supplemental Material [37]).

Zero energy Majorana end modes in a semi-infinite chain.-We first consider the case of a semi-infinite chain extending to $+\infty$ with a single boundary at $j=1$. The definition, Eq. (3), of the generating function gives that in the case of an end state, $G(z)$ only diverges exponentially as $z$ approaches the singular points $z_{i}$ that satisfy $\left|z_{i}\right|>1$. Hence, all zeros $z_{i}$ of $\operatorname{det}(K+z M)$ in the denominator of Eq. (4) with $\left|z_{i}\right|<1$ or $\left|z_{i}\right|=1$ need to be canceled, along with the explicit $z=1$ pole in Eq. (4). Applying these conditions to the generating function for $\mu<J$ gives a Majorana end state localized at $j=1$ with energy $E=0$ and wave function

$$
\psi_{j} \propto z_{1}^{-j}\left(\begin{array}{c}
e^{-i \theta} \\
1
\end{array}\right)
$$

where $\theta$ is the phase of the superconducting coupling $\Delta$ and $z_{1}=1-(2 J / \mu)$.

Finding zero energy modes is a manifestation of the system being in a topological phase. This is a surprising result given that the presence of the nonlocal couplings dramatically alters the notion of locality and dimensionality. 
We will later connect the presence of the zero modes with the nontrivial value of a topological invariant that characterizes the topological phase of the nonlocal chain.

The zero energy Majorana end state, Eq. (5), of the semiinfinite chain is the unique, spectrally isolated state, which is both an eigenstate of the Hamiltonian and of the particlehole operator $\mathcal{P}=\mathcal{K} \sigma_{2}$, where $\mathcal{K}$ is complex conjugation and $\sigma_{2}$ the second Pauli matrix. This property pins the Majorana end state to $E=0$. If the system is deformed away from the exactly solvable point by a series of unitary operations (local or not) that commute with particle-hole symmetry, this property is preserved as long as the energy gap does not close. Hence, the end mode, Eq. (5), is topologically stable in the same way as the end modes of the local Kitaev chain [30].

Gapped end modes in a finite chain.-We now turn to the case of a finite chain of $N$ sites with two boundaries, as shown in Fig. 1 (top). From this fully connected graph it is possible to distinguish its two ends through the pairing terms of Hamiltonian (1), that naturally encodes a direction. This is apparent when we rewrite the Hamiltonian using Majorana operators, as shown in Fig. 1 (bottom). In addition to the exponential singularities at $z_{i}$ for $\left|z_{i}\right|>1$ that we met in the semi-infinite case, the end state generating function of a finite chain also diverges linearly with the number of sites $N$ at $z=1$ (see Supplemental Material [37]).

To proceed, we first consider the symmetries of Hamiltonian (1) with two boundaries that are absent on a semi-infinite chain. These symmetries, present also in a local chain, impose additional conditions that are useful to analytically determine the nonzero energy of the end modes. We observe that a reflection of the chain about its center, $j \rightarrow N-j+1$ with $l \rightarrow-l$, together with a substitution of $a_{j} \rightarrow-a_{j}, a_{j}^{\dagger} \rightarrow a_{j}^{\dagger}$ transforms the Hamiltonian as $H \rightarrow-H$, that give $\psi_{j}^{A}=-\psi_{N-j+1}^{A}$ and $\psi_{j}^{B}=\psi_{N-j+1}^{B}$. A second set of possible transformations, including a reflection along with $a_{j} \rightarrow a_{j}$ and $a_{j}^{\dagger} \rightarrow-a_{j}^{\dagger}$, that also transform the Hamiltonian as $H \rightarrow-H$, gives $\psi_{j}^{A}=\psi_{N-j+1}^{A}$ and $\psi_{j}^{B}=-\psi_{N-j+1}^{B}$. These conditions impose either $\phi_{1}=\sum_{j}^{N} \psi_{j}^{A}=0$ or $\phi_{2}=\sum_{j}^{N} \psi_{j}^{B}=0$, respectively.

We first set $\phi_{2}=0$ in Eq. (4). As we have seen above, demanding that the generating function describes an exponentially localized mode imposes conditions on its divergence properties. These conditions determine that, for $\mu<J$, the energy of the mode is given by

$$
E_{+}=\frac{J-\mu}{2} \frac{J^{2}-|\Delta|^{2}}{J^{2}+|\Delta|^{2}}
$$

and the amplitudes of the corresponding state are given by

$$
\psi_{j}^{+} \propto z_{1}^{-j}\left(\begin{array}{c}
1 \\
\frac{J}{\Delta^{*}}
\end{array}\right)+z_{1}^{j-N-1}\left(\begin{array}{c}
1 \\
-\frac{J}{\Delta^{*}}
\end{array}\right)
$$

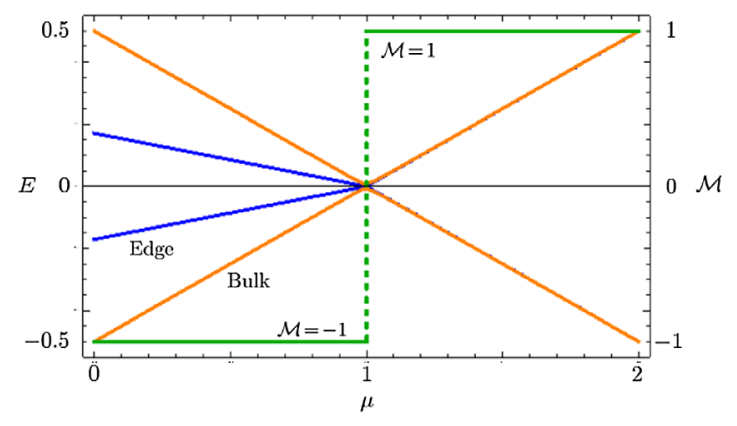

FIG. 2. The topological behavior of the open chain as a function of the chemical potential $\mu$ for $J=1, \Delta=0.7$, and $N=100$. The system undergoes a topological phase transition at $\mu=J$. For $\mu<J$ the end states (blue) are energetically distinct from the lowest bulk states (orange), while they coincide for $\mu>J$. The bulk gap closes as $N \rightarrow \infty$ at $\mu=J$. The sign, $\mathcal{M}$, of the Pfaffian (green) identifies the topological $\mathcal{M}=-1$ from the nontopological $\mathcal{M}=+1$ regimes of the open chain.

where $z_{1}=\left(2 E_{+}+2 J-\mu\right) /\left(2 E_{+}-\mu\right)$ is the inverse of the canceled pole. When we set $\phi_{1}=0$, we obtain the second energy, $E_{-}=-E_{+}$, with the mode given by $\psi_{j}^{-} \propto\left[\sigma_{1} \psi_{j}^{+}\right]^{*}$. Hence, the localized Majorana end modes of a finite chain have in general nonzero energies $E_{ \pm}$determined by Eq. (6) that are independent of the system size $N$.

The nonzero value of the energies $E_{ \pm}$is due to the nonlocal coupling between the end modes at the two edges regardless of their distance. These couplings hybridize the two Majorana end modes making them have support simultaneously at both ends, as it is apparent from Eq. (7). This is equivalent to the hybridization of overlapping end modes in local Hamiltonians, though now this effect cannot be removed by increasing the size $N$ of the system. Note that the special case with $J= \pm|\Delta|$ and $\mu=0$ gives rise to end Majorana modes with $E_{ \pm}=0$ and amplitudes $\psi_{j}^{ \pm}$that are ultralocalized at the ends of the chain, as expected from the Majorana description given in Fig. 1 (bottom).

The presence of end modes can also be diagnosed by a topological index characterizing the phase of the system. Hamiltonian (1) belongs to symmetry class $\mathrm{D}$ in the classification of $[38,39]$. Both in $d=1$ and $d=0$, this symmetry class has a $\mathbb{Z}_{2}$ topological classification. In both cases the topological invariant is a parity, $\mathcal{M}$, that can be evaluated for nondegenerate open or closed chains in terms of the Pfaffian of the corresponding Hamiltonian matrix. Hence, it can be used directly for the case of the infiniterange Hamiltonian with $\alpha=0$ [40]. By varying the chemical potential $\mu$ we observe that the parity becomes nontrivial, $\mathcal{M}=-1$, for $\mu<J$, as shown in Fig. 2. In that regime the end states, while they have nonzero energy, are energetically distinct from the bulk states. This is in contrast to the nontopological regime, $\mathcal{M}=+1$, where the end states cease to exist. 


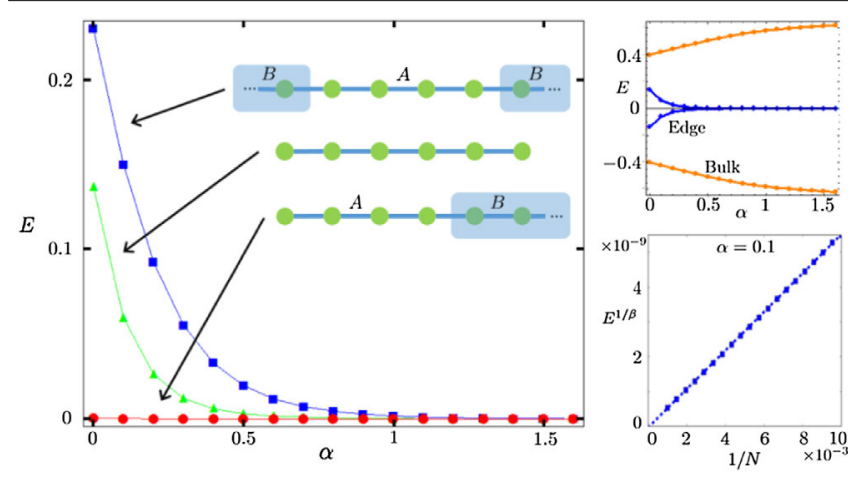

FIG. 3. With $J=1, \Delta=0.7, \mu=0.2$, and $N=400$. (Left) Energies of the Majorana end modes for a chain with power law couplings parametrized by $\alpha$, corresponding to the physical spectrum (green), the entanglement spectrum with two cuts (blue) and the entanglement spectrum with one cut (red) for $A$ of size $N_{A}=204$. The relevant chains with their partitions for the entanglement spectra are also depicted. (Right top) Ground state (blue) and first excited state (orange) for various $\alpha$. (Right bottom) The end state energy, linearized with $1 / \beta$, as a function of system size for $\alpha=0.1, \beta=0.137$.

The topological phase of the nonlocal model is robust to weak disorder in the chemical potential. In this case, the Majorana end modes remain exponentially localized and the Majorana number, $\mathcal{M}$, remains nontrivial, as in the case with no disorder.

Power law behavior.-We now show that the characteristics of the infinite-range model are robust when the couplings, in Eq. (1), take the more physical power law form. To demonstrate this we perform a numerical treatment of a finite chain for various $\alpha$. Even though a real spectrum of the semi-infinite chain cannot be obtained numerically, we show that, rather surprisingly, its properties are manifested in the entanglement spectrum [41]. This spectrum is the set of eigenvalues of the entanglement Hamiltonian $H_{\mathrm{E}}=-\ln \rho_{A}$, defined through the reduced density matrix $\rho_{A}=\operatorname{tr}_{B}|\Psi\rangle\langle\Psi|$ for a chain bipartitioned into region $A$ and its complement $B$, where $|\Psi\rangle$ is the manybody ground state of the system [42]. Unlike the real spectrum of a finite chain, that has necessarily two ends, a choice of region $A$ can give rise to a single virtual boundary. In this case the entanglement spectrum hosts degenerate zero modes, indicating the presence of Majorana end modes in the same way as a local chain. Figure 3 shows that this holds for any $\alpha$. Thus, the topological phase can be diagnosed with the same tools and criteria as a local chain.

The zero energy entanglement mode is in agreement with the analytical solution of the semi-infinite case with $\alpha=0$ derived in the previous section, thus demonstrating the robustness of the $E=0$ states to finite range couplings. It also demonstrates the physical relevance of the semiinfinite chain as its spectrum is manifested in the correlations of the ground state of a finite system. Interestingly, the entanglement eigenstate has a power-law decay, instead of the exponential decay which we obtained in Eq. (5). This is a manifestation of the nonlocal couplings on the correlations across the partition (see Supplemental Material [37]). For a bipartition in which the two regions interface at two separated points, the system is described by an open finite entanglement Hamiltonian. In this case, the spectral degeneracy is lifted due to the long-range coupling between the edge states.

The energy splitting of the Majorana end states due to the infinite-range interactions remains present even if we employ decaying couplings with $\alpha \neq 0$. As $\alpha$ varies, there is a continuous interpolation between the finite energy $E_{+}$ given in Eq. (6) at $\alpha=0$ and the zero energy at $\alpha \rightarrow \infty$. As one would expect from the argument of hybridization, the energy will decay with $1 / N^{\beta}$, where $\beta \approx \alpha$ and depends on the coupling parameters as well as $\alpha$. This property is shown in Fig. 3 for both the real and the entanglement spectra. Similar robustness in the values of $\alpha$ is exhibited by the topological index $\mathcal{M}$. As $\alpha$ increases, the topological index preserves its nontrivial value $\mathcal{M}=-1$ for all $\alpha$ when $\mu<J$. As can be seen in Fig. 3 (right top), the limiting cases of $\alpha=0$ and $\alpha \rightarrow \infty$ are adiabatically connected in the topological sector.

When $\alpha \neq 0$ the interaction between the end modes decays as their distance increases due to the power law dependence of the couplings. This causes the energy of the modes to tend to zero for increasing $N$, as shown in Fig. 3 (right bottom). Nevertheless, for small enough $\alpha$ and $N$ the characteristic of nonzero energy will be present as the couplings will effectively have infinite range. These results remain qualitatively the same when the tunneling couplings are assigned a shorter range interaction compared to the pairing ones.

Conclusions.-Our analytical treatment demonstrates that the infinite-range Kitaev chain is topological. It supports Majorana zero modes and it has a nontrivial topological index. The zero modes are exact in semiinfinite chains; a finite chain, regardless of its size, gives hybridized Majorana end modes with nonzero energy. This result is derived exactly, presenting a theoretical framework for solving other long range problems. Importantly, the Majorana zero modes are identified in the entanglement spectrum of finite systems, for an appropriate partition. Thus, it presents a diagnostic tool for numerical simulations or experimental implementations of nonlocal models. The infinite range 1D Majorana chain is an archetype model against which other models can now be compared analytically.

Notably, long-range interactions become increasingly relevant in current theoretical and experimental investigations. On the experimental front, Rydberg atoms, trapped ions, and atoms in optical cavities can be designed with interactions of arbitrary range. Many of these systems are engineered as spin chains, which can be mapped to long range fermionic chains [2,15]. Alternatively, 
Shiba chains provide a solid-state system that naturally supports nonlocal interactions [25-29]. Here, magnetic impurities placed on an $s$-wave superconductor have an effective Hamiltonian that contains long range hopping and pairing couplings. On the theoretical front, we envision that the unique properties of nonlocal models can help us understand exotic effects of strongly correlated systems $[43,44]$.

We would like to thank Hans Peter Büchler, Steve Simon, and Chris Turner for inspiring conversations. This work was supported in part by the Engineering and Physical Sciences Research Council Grant No. EP/I038683/1.

\section{*py11kp@leeds.ac.uk}

[1] X. Li and S. Das Sarma, Nat. Commun. 6, 7137 (2015).

[2] R. Islam, C. Senko, W. C. Campbell, S. Korenblit, J. Smith, A. Lee, E. E. Edwards, C.-C. J. Wang, J. K. Freericks, and C. Monroe, Science 340, 583 (2013).

[3] Y. Li, L. He, and W. Hofstetter, Phys. Rev. A 87, 051604 (2013).

[4] J. Bardeen, L. N. Cooper, and J. R. Schrieffer, Phys. Rev. 106, 162 (1957).

[5] R. B. Laughlin, Phys. Rev. Lett. 50, 1395 (1983).

[6] J. K. Asbóth, H. Ritsch, and P. Domokos, Phys. Rev. Lett. 98, 203008 (2007).

[7] W. Fu and S. Sachdev, Phys. Rev. B 94, 035135 (2016).

[8] R. Landig, L. Hruby, N. Dogra, M. Landini, R. Mottl, T. Donner, and T. Esslinger, Nature (London) 532, 476 (2016).

[9] S. R. Manmana, E. M. Stoudenmire, K. R. A. Hazzard, A. M. Rey, and A. V. Gorshkov, Phys. Rev. B 87, 081106 (2013).

[10] S. Sachdev and J. Ye, Phys. Rev. Lett. 70, 3339 (1993).

[11] S. A. Cannas, Phys. Rev. B 52, 3034 (1995).

[12] J. L. Monroe, J. Phys. A 31, 9809 (1998).

[13] M. Barati and A. Ramazani, Phys. Rev. B 64, 024407 (2001).

[14] L. Arrachea and M. J. Rozenberg, Phys. Rev. B 65, 224430 (2002).

[15] D. Porras and J. I. Cirac, Phys. Rev. Lett. 92, 207901 (2004).

[16] P. Fendley, J. Stat. Mech. (2012) P11020.

[17] Z.-X. Gong, M. F. Maghrebi, A. Hu, M. Foss-Feig, P. Richerme, C. Monroe, and A. V. Gorshkov, Phys. Rev. B 93, 205115 (2016).

[18] Z.-X. Gong, M. F. Maghrebi, A. Hu, M. L. Wall, M. FossFeig, and A. V. Gorshkov, Phys. Rev. B 93, 041102 (2016).

[19] S. M. Giampaolo, K. Simonov, A. Capolupo, and B. C. Hiesmayr, arXiv:1607.05692.

[20] W. DeGottardi, M. Thakurathi, S. Vishveshwara, and D. Sen, Phys. Rev. B 88, 165111 (2013).
[21] D. Vodola, L. Lepori, E. Ercolessi, A. V. Gorshkov, and G. Pupillo, Phys. Rev. Lett. 113, 156402 (2014).

[22] D. Vodola, L. Lepori, E. Ercolessi, and G. Pupillo, New J. Phys. 18, 015001 (2016).

[23] M. Van Regemortel, D. Sels, and M. Wouters, Phys. Rev. A 93, 032311 (2016).

[24] O. Viyuela, D. Vodola, G. Pupillo, and M. A. MartinDelgado, Phys. Rev. B 94, 125121 (2016).

[25] F. Pientka, L. I. Glazman, and F. von Oppen, Phys. Rev. B 88, 155420 (2013).

[26] F. Pientka, L. I. Glazman, and F. von Oppen, Phys. Rev. B 89, 180505(R) (2014).

[27] F. Pientka, Y. Peng, L. Glazman, and F. von Oppen, Phys. Scr. T164, 014008 (2015).

[28] P. M. R. Brydon, S. Das Sarma, H.-Y. Hui, and J. D. Sau, Phys. Rev. B 91, 064505 (2015).

[29] J. Röntynen and T. Ojanen, Phys. Rev. Lett. 114, 236803 (2015).

[30] A. Kitaev, Phys. Usp. 44, 131 (2001).

[31] J. K. Pachos and M. B. Plenio, Phys. Rev. Lett. 93, 056402 (2004).

[32] A. Kay, D. K. K. Lee, J. K. Pachos, M. B. Plenio, M. E. Reuter, and E. Rico, Opt. Spectrosc. 99, 339 (2005).

[33] S. S. Pershoguba and V. M. Yakovenko, Phys. Rev. B 86, 075304 (2012).

[34] D. C. Sticlet, Ph.D. thesis, Universite Paris-SudXI, 2012.

[35] K. Meichanetzidis, M. Cirio, J. K. Pachos, and V. Lahtinen, Phys. Rev. B 94, 115158 (2016).

[36] I. M. Gelfand and A. Shen, Algebra (Birkhäuser, Basel, 1993), p. 102.

[37] See Supplemental Material at http://link.aps.org/ supplemental/10.1103/PhysRevLett.118.267002 for detailed calculations.

[38] A. P. Schnyder, S. Ryu, A. Furusaki, and A. W. W. Ludwig, Phys. Rev. B 78, 195125 (2008).

[39] A. Kitaev, AIP Conf. Proc. 1134, 22 (2009).

[40] Note that it is possible to assign a $\mathbb{Z}$ classification to the model by defining a local chiral symmetry. For a short range chain the model would then belong to the BDI symmetry class. However, terms may be added to the Hamiltonian that break this chiral symmetry, so that the chain remains in class D. This $\mathbb{Z}_{2}$ classication sufficiently describes the topological phase of the chain and can be accurately probed by the Pfaffian invariant.

[41] H. Li and F. D. M. Haldane, Phys. Rev. Lett. 101, 010504 (2008).

[42] M. Legner and T. Neupert, Phys. Rev. B 88, 115114 (2013).

[43] C. J. Turner, K. Meichanetzidis, Z. Papic, and J. K. Pachos, Nat. Commun. 8, 14926 (2017).

[44] S. Gangadharaiah, B. Braunecker, P. Simon, and D. Loss, Phys. Rev. Lett. 107, 036801 (2011). 